\title{
Method of Forming Stable States of Dense High-Temperature Plasma
}

\author{
Stanislav I. Fisenko, Igor S. Fisenko \\ Rusthermosynthesis, Moscow, Russia \\ Email: stanislavfisenko@yandex.ru \\ Received January 26, 2013; revised February 25, 2013; accepted March 7, 2013
}

Copyright (C) 2013 Stanislav I. Fisenko, Igor S. Fisenko. This is an open access article distributed under the Creative Commons Attribution License, which permits unrestricted use, distribution, and reproduction in any medium, provided the original work is properly cited.

\begin{abstract}
The concept of gravitational radiation as a radiation of one level with the electromagnetic radiation is based on theoretically proved and experimentally confirmed fact of existence of electron's stationary states in own gravitational field, characterized by gravitational constant $K=10^{42} G(G$-Newtonian gravitational constant) and by irremovable space-time curvature. The received results strictly correspond to principles of the relativistic theory of gravitation and the quantum mechanics. The given work contributes into further elaboration of the findings considering their application to dense high-temperature plasma of multiple-charge ions. This is due to quantitative character of electron gravitational radiation spectrum such that amplification of gravitational radiation may take place only in multiple-charge ion high-temperature plasma. In elaboration of the authors' works [1-4], an essential instantiation of the concept of fusion plasma's steady states formation (as the last paragraph outlines) and boundary conditions refinement in the electron's stationarystates-in-proper-gravitational-field problem are appended to this article.
\end{abstract}

Keywords: Gravity; Electron; Spectrum; Discharge; Fusion

\section{Gravitational Radiation as a Radiation of the Same Level as Electromagnetic}

For a mathematical model of interest, which describes a banded spectrum of stationary states of electrons in the proper gravitational field, two aspects are of importance. First, in Einstein's field equations $\kappa$ is a constant which relates the space-time geometrical properties with the distribution of physical matter, so that the origin of the equations is not connected with the numerical limitation of the $\kappa$ value. Only the requirement of conformity with the Newtonian Classical Theory of Gravity leads to the small value $\kappa=8 \pi G / c^{4}$, where $G, c$ are, respectively, the Newtonian gravitational constant and the velocity of light. Such requirement follows from the primary concept of the Einstein General Theory of Relativity (GR) as a relativistic generalization of the Newtonian Theory of Gravity. Second, the most general form of relativistic gravitation equations are equations with the $\Lambda$ term. The limiting transition to weak fields leads to the equation

$$
\Delta \Phi=-4 \pi \rho G+\Lambda c^{2},
$$

where $\Phi$ is the field scalar potential, $\rho$ is the source density. This circumstance, eventually, is crucial for neglecting the $\Lambda$ term, because only in this case the GR is a generalization of the Classical Theory of Gravity. Therefore, the numerical values of $\kappa=8 \pi G / c^{4}$ and $\Lambda=0$ in the GR equations are not associated with the origin of the equations, but follow only from the conformity of the GR with the classical theory.

From the 70's onwards, it became obvious [5] that in the quantum region the numerical value of $G$ was not compatible with the principles of quantum mechanics. The essence of the problem of the generalization of relativistic equations on the quantum level was thus outlined: such generalization must match the numerical values of the gravity constants in the quantum and classical regions.

In the development of these results, as a micro-level approximation of Einstein's field equations, a model is proposed, based on the following assumption $[1,2]$ :

"The gravitational field within the region of localization of an elementary particle having a mass $m_{0}$ is characterized by the values of the gravity constant $K$ and of the constant $\Lambda$ that lead to the stationary states of the particle in its proper gravitational field, and the particle stationary states as such are the sources of the gravitational field with the Newtonian gravity constant G."

The most general approach in the Gravity Theory is 
the one which takes torsion into account and treats the gravitational field as a gauge field, acting on equal terms with other fundamental fields [6].

Complexity of solving this problem compels us to employ a simpler approximation, namely: energy spectrum calculations in a relativistic fine-structure approximation. In this approximation the problem of the stationary states of an elementary source in the proper gravitational field will be reduced to solving the following equations:

$$
\begin{aligned}
& f^{\prime \prime}+\left(\frac{v^{\prime}-\lambda^{\prime}}{2}+\frac{2}{r}\right) f^{\prime}+\mathrm{e}^{\lambda}\left(K_{n}^{2} \mathrm{e}^{-v}-K_{0}^{2}-\frac{l(l+1)}{r^{2}}\right) f=0 \\
& -\mathrm{e}^{-\lambda}\left(\frac{1}{r^{2}}-\frac{\lambda^{\prime}}{r}\right)+\frac{1}{r^{2}}+\Lambda \\
& =\beta(2 l+1)\left\{f^{2} \mathrm{e}\left[\mathrm{e}^{-\lambda} K_{n}^{2}+K_{0}^{2}+\frac{l(l+1)}{r^{2}}\right]+f^{\prime 2} \mathrm{e}^{-\lambda}\right\} \\
& -\mathrm{e}^{-\lambda}\left(\frac{1}{r^{2}}+\frac{v^{\prime}}{r}\right)+\frac{1}{r^{2}}+\Lambda \\
& =\beta(2 l+1)\left\{f^{2}\left[K_{0}^{2}-K_{n}^{2} \mathrm{e}^{-v}+\frac{l(l+1)}{r^{2}}\right]-\mathrm{e}^{\lambda} f^{\prime 2}\right\} \\
& \left\{-\frac{1}{2}\left(v^{\prime \prime}+v^{\prime 2}\right)-\left(v^{\prime}+\lambda^{\prime}\right)\left(\frac{v^{\prime}}{4}+\frac{1}{r}\right)+\frac{1}{r^{2}}\left(1+\mathrm{e}^{\lambda}\right)\right\}_{r=r_{n}}=0 \\
& f\left(\sqrt{\Lambda^{-1}}\right)=0 \\
& f\left(r_{n}\right)=0 \\
& \lambda(0)=v(0)=0 \\
& \int_{0}^{r_{n}} f^{2} r^{2} \mathrm{~d} r=1
\end{aligned}
$$

Equations (1)-(3) follow from Equations (9) and (10)

$$
\begin{gathered}
\left\{-g^{\mu v} \frac{\partial}{\partial x_{\mu}} \frac{\partial}{\partial x_{v}}+g^{\mu v} \Gamma_{\mu v}^{\alpha} \frac{\partial}{\partial x_{\alpha}}-K_{0}^{2}\right\} \Psi=0 \\
R_{\mu v}-\frac{1}{2} g_{\mu v} R=-\kappa\left(T_{\mu v}-\mu g_{\mu v}\right),
\end{gathered}
$$

after the substitution of $\Psi$ in the form of $\Psi=f_{E l}(r) Y_{l m}(\theta, \varphi) \exp \left(\frac{-\mathrm{i} E t}{\hbar}\right)$ into them and specific computations in the central-symmetry field metric with the interval defined by the expression [7]

$$
\mathrm{d} S^{2}=c^{2} \mathrm{e}^{v} \mathrm{~d} t^{2}-r^{2}\left(\mathrm{~d} \theta^{2}+\sin ^{2} \theta \mathrm{d} \varphi^{2}\right)-\mathrm{e}^{\lambda} \mathrm{d} r^{2}
$$

The following notation is used above: $f_{E l}$ is the radial wave function that describes the states with a defi- nite energy $E$ and the orbital moment $l$ (hereafter the subscripts $E l$ are omitted), $Y_{l m}(\theta, \varphi)$-are spherical functions, $K_{n}=E_{n} / \hbar c, K_{0}=c m_{0} / \hbar, \beta=(\kappa / 4 \pi)\left(\hbar / m_{0}\right)$.

Condition (4) defines $r_{n}$, whereas Equations (10) through (7) are the boundary conditions and the normalization condition for the function $f$, respectively. Condition (4) in the general case has the form $R\left(K, r_{n}\right)=$ $R\left(G, r_{n}\right)$. Neglecting the proper gravitational field with the constant $G$, we shall write down this condition as $R\left(K, r_{n}\right)=0$, to which equality (4) actually corresponds.

The right-hand sides of Equations (2) and (3) are calculated basing on the general expression for the energymomentum tensor of the complex scalar field:

$$
T_{\mu v}=\Psi_{, \mu}^{+} \Psi_{, v}+\Psi_{, v}^{+} \Psi_{, \mu}-\left(\Psi_{, \mu}^{+} \Psi^{, \mu}-K_{0}^{2} \Psi^{+} \Psi\right)
$$

The appropriate components $T_{\mu v}$ are obtained by summation over the index $m$ with application of characteristic identities for spherical functions [8] after the substitution of $\Psi=f(r) Y_{l m}(\theta, \varphi) \exp \left(\frac{-\mathrm{i} E t}{\hbar}\right)$ into (12).

In its simplest approximation (from the point of view of the original mathematical estimates) the problem on steady states in proper gravitational field (with constants $K$ and $\Lambda$ ) is solved by [1]. The solution of this problem provides the following conclusions.

1) With the numeric values $K \approx 5.1 \times 10^{31} \mathrm{~N} \cdot \mathrm{m}^{2} \cdot \mathrm{kg}^{-2}$ and $\Lambda=4.4 \times 10^{29} \mathrm{~m}^{-2}$, there is a spectrum of steady states of the electron in proper gravitational field $(0.511$ $\mathrm{MeV}-0.681 \mathrm{MeV})$. The basic state is the observed electron rest energy $0.511 \mathrm{MeV}$.

2) These steady states are the sources of the gravitational field with the $G$ constant.

3) The transitions to stationary states of the electron in proper gravitational field cause gravitational emission, which is characterized by constant $K$, i.e. gravitational emission is an emission of the same level as electromagnetic (electric charge $e$, gravitational charge $m \sqrt{K}$ ). In this respect there is no point in saying that gravitational effects in the quantum area are characterized by the $G$ constant, as this constant belongs only to the macroscopic area and cannot be transferred to the quantum level (which is also evident from the negative results of registration of gravitational waves with the $G$ constant, they do not exist).

Existence of such numerical value $\Lambda$ denotes a phenomenon having a deep physical sense: introduction into density of the Lagrange function of a constant member independent on a state of the field. This means that the time-space has an inherent curving which is connected with neither the matter nor the gravitational waves. The distance at which the gravitational field with the constant $K$ is localized is less than the Compton wavelength, and for the electron, for example, this value is of the order of 
its classical radius. At distances larger than this one, the gravitational field is characterized by the constant $G$, i.e., correct transition to Classical GR holds.

\section{Spectral Lines Widening of the Radiation of Multiple-Charge Ions}

Figures 1 and 2 show characteristic parts of micropinch soft X-ray radiation spectrum. Micropinch spectrum line widening does not correspond to existing electromagnetic conceptions but corresponds to such plasma thermodynamic states which can only be obtained with the help of compression by gravitational field [2,3], radiation flashes of which takes place during plasma thermalization in a discharge local space. Such statement is based on the comparison of experimental and expected parts of the spectrum shown in Figures 2(a) and (b). Adjustment of the expected spectrum portion to the experimental one (see [1]) was made by selecting average values of density $\rho$, electron temperature Te and velocity gradient $U$ of the substance hydrodynamic motion.

As a mechanism of spectrum lines widening, a Doppler, radiation and impact widening were considered. Such adjustment according to said widening mechanisms does not lead to complete reproduction of the registered part of the micropinch radiation spectrum. This is the evidence (under the condition of independent conformation of the macroscopic parameters adjustment) of additional widening mechanism existence due to electron excited states and corresponding gravitational radiation spectrum part already not having clearly expressed lines because of energy transfer in the spectrum to the longwave area.

That is to say that the additional mechanism of spectral lines widening of the characteristic electromagnetic radiation of multiple-charge ions (in the conditions of plasma compression by radiated gravitational field) is the

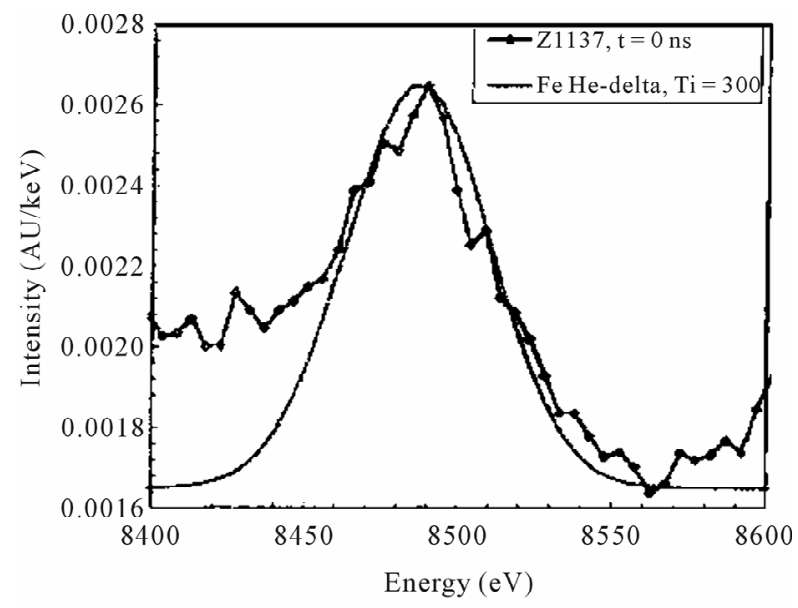

Figure 1. Measured Fe He- $\delta$ line at $8.488 \mathrm{keV}$ (broken curve) compared to calculation (smooth curve), [9].

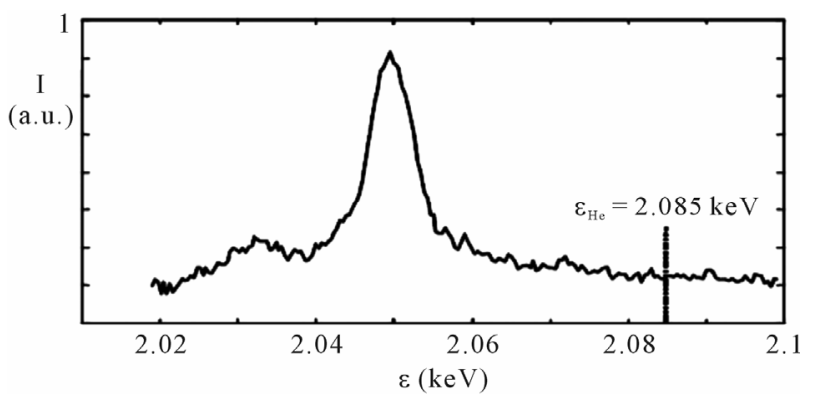

(a)

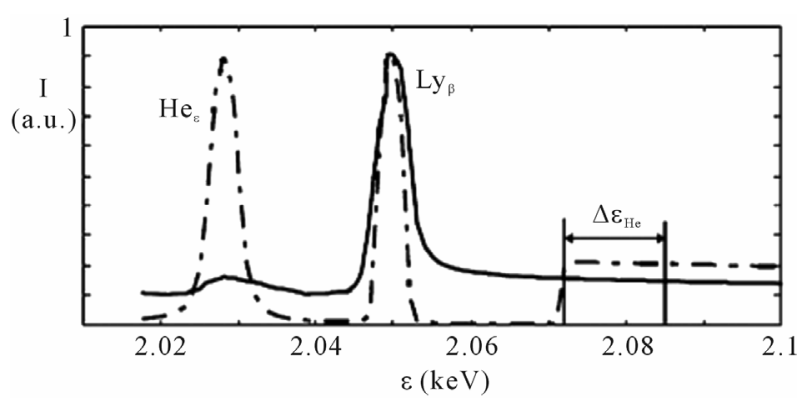

(b)

Figure 2. Experimental (a) and calculated (b) parts of a micropinch spectrum normalized for line $\mathrm{Ly}_{\beta}$ intensity in the area of the basic state ionization threshold of He-like ions. The firm line in variant (b) corresponds to density of $0.1 \mathrm{~g} / \mathrm{cm}^{3}$, the dotted line-to $0.01 \mathrm{~g} / \mathrm{cm}^{3}$; it was assumed that $T_{e}=0.35 \mathrm{keV}$ (see [1]).

only and unequivocal way of quenching electrons excited states at the radiating energy levels of ions and exciting these levels by gravitational radiation at resonance frequencies. Such increase in probability of ion transitions in other states results in additional spectral lines widening of the characteristic radiation. The reason for quick degradation of micropinches in various pulse high-currency discharges with multiple-charge ions is also clear. There is only partial thermolization of accelerated plasma with the power of gravitational radiation not sufficient for maintaining steady states [4].

\section{Thermonuclear Plasma Steady States Generation}

The problem of controlled fusion realization is directly connected to obtaining steady state of dense high-temperature plasma.

It can also be unambiguously stated that the present state of the art (retaining plasma by magnetic fields of various configurations, squeezing by laser radiation) does not solve the problem of dense high-temperature plasma retention for a time required for the reaction of nuclear fusion but only solving the problem of heating plasma to the state when these reactions can exist. In the offered method of forming dense-high temperature plasma steady states for nuclear fusion a new fundamental concept is 
used, namely retaining plasma by radiated gravitational field as radiation of the same kind as electromagnetic:

Forming and accelerating binary plasma with multivalent ions by accelerating magnetic field in a pulse high-current discharge.

Injection of binary plasma from the space of the accelerating magnetic field:

Exciting stationary states of an electron in its own gravitational field in the range of energy up to $171 \mathrm{keV}$ with following radiation under the condition of quenching lower excited energy levels of ion electron shell of a heavy component (including quenching excited state of electrons directly in nuclei of small sequential number as carbon) when retarding plasma bunch ejected from the space of the accelerating magnetic field. Cascade transitions from the upper levels are realized in the process of gravitational radiation energy transit to long-wave range.

The sequence of the operations is carried out in a twosectional chamber of MAGO installation (Figure 3, developed in Experimental Physics Research Institute, Sarov, [10]; the structure of the installation is most suitable for the claimed method of forming steady states of the dense high-temperature plasma) with magnetodynamic outflow of plasma and further conversion of the plasma bunch energy (in the process of quenching) in the plasma heat energy for securing both further plasma heating and exciting gravitational radiation and its transit into a long-wave part of the spectrum with consequent plasma compression in the condition of radiation blocking and increasing [4].

Of interest, there are two modes of the installation operations depending on the work gas composition [11].

1) A composition with hydrogen and (for example) xenon providing for achieving steady states of plasma with consequent realization of thermonuclear reactions for compositions of $(d+t)+$ multi-charge atoms type.

Fusion reaction creating helium generates neutrons

$$
{ }_{1}^{2} \mathrm{H}+{ }_{1}^{3} \mathrm{H} \rightarrow{ }_{2}^{4} \mathrm{He}+{ }_{0}^{1} n+17.6 \mathrm{MeV}
$$

and was embodied in the well-known Teller-Ulam design with radiation implosion.

An application of the compression-by-the-radiatedgravitational-field design, unlike the Teller-Ulam design, is not limited by the minimum discharge power attendant to the usage of a plutonium nucleus. This means both a feasibility of this fast fusion reaction a steady mode for low-power discharges, and (under certain conditions) a feasibility of explosive energy release without the use of fissile elements (like plutonium and uranium) for highpower discharges.

2) A composition with hydrogen and carbon providing thermonuclear reactions of carbon cycle in plasma steady

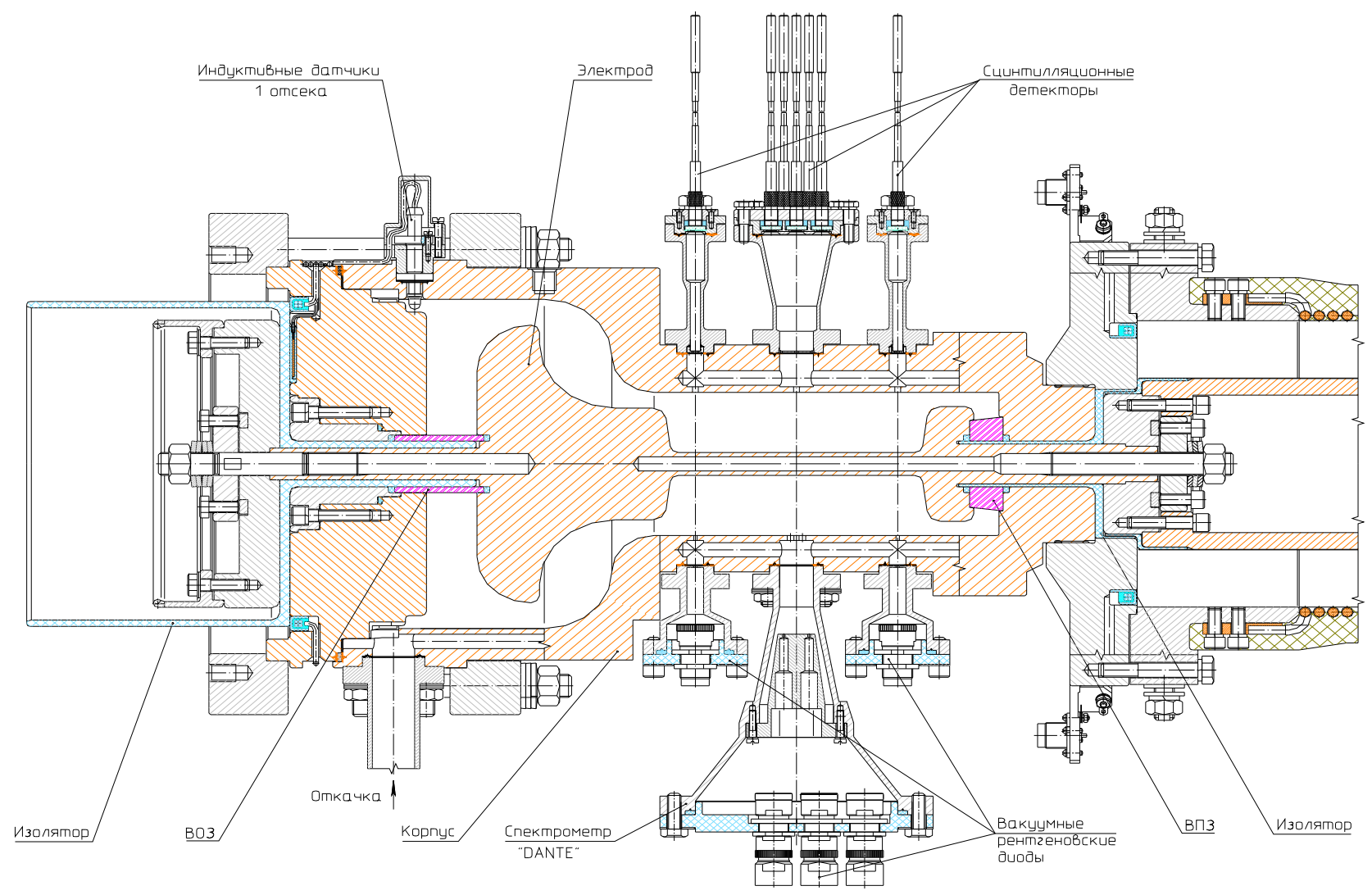

Figure 3. Outline drawing of the discharge chamber (MAGO chamber) and X-ray diagnostic system. 
state mode, including energy pick-up in the form of electromagnetic radiation energy

$\mathrm{CNO}$ cycle is a set of fusion reactions resulting in conversion of hydrogen into helium using carbon as a catalyst.

In a compact notation, this cycle would be written as

$$
\begin{aligned}
& { }^{12} \mathrm{C}(\mathrm{p}, \gamma){ }^{13} \mathrm{~N}\left(\mathrm{e}^{+} v\right){ }^{13} \mathrm{C}(\mathrm{p}, \gamma){ }^{14} \mathrm{~N}(\mathrm{p}, \gamma) \\
& { }^{15} \mathrm{O}\left(\mathrm{e}^{+} v\right){ }^{15} \mathrm{~N}(\mathrm{p}, \alpha){ }^{12} \mathrm{C}
\end{aligned}
$$

The ${ }^{15} \mathrm{~N}(\mathrm{p}, \alpha){ }^{12} \mathrm{C}$ reaction rounds the cycle out. The net result is that four protons turn into an $\alpha$-particle${ }^{4} \mathrm{He}$ nucleus with no neutrons among end products of the cycle. Producing one helium nucleus releases $25 \mathrm{MeV}$, the produced neutrinos carry away about $5 \%$ more of that energy. A peculiarity of such fusion reaction cycle is that it occurs in natural conditions of astrophysical objects. At the same time, e.g. the design features of MAGO facility allow achieving such a volt-ampere characteristics (VAC) mode that boosts the speed of carbon cycle reactions. An implementation of carbon cycle in the gravitational compression design may possibly become a basic design to form steady states of the thermonuclear plasma, bearing in mind low abundance of hydrogen and lithium isotopes that react with no neutrons being produced either.

\section{REFERENCES}

[1] S. I. Fisenko and I. S. Fisenko, "Gravitational Interaction on Quantum Level and Consequences Thereof," The Old and New Concepts of Physics, Vol. 6, No. 4, 2009, pp. 495-521.
[2] S. I. Fisenko and I. S. Fisenko, "The Discrete Energy Spectrum of the Gravitational Radiation in the Relativistic Theory of Gravitation," International Journal of Theoretical and Applied Physics (IJTAP), Vol. 2, No. 2, 2012, pp. 32-39.

[3] S. I. Fisenko and I. S. Fisenko, "Concept of a Thermonuclear Reactor on the Principle of Gravitatational Confinement of High-Temperature Plasma," Applied Physics Research, Vol. 2, No. 2, 2010, pp. 71-77.

[4] S. I. Fisenko and I. S. Fisenko, "Gravitational Radiation of the Relativistic Theory of Gravitation," British Journal of Science, Vol. 2, No. 1, 2011, pp. 1-20.

[5] C. Siravam and K. Sinha, "The Concept of 'Strong' Gravity,” Physics Reports, Vol. 51, No. 3, 1979, pp. 112-123.

[6] D. D. Ivanenko, et al., "Gauge Theory of Gravity," MGU Publishing House, Moscow, 1985, pp. 71-73.

[7] L. D. Landau and E. M. Lifshitz, "Field Theory," Publishing House "Nauka", Moscow, 1976, pp. 451-457.

[8] D. A. Warshalovich, et al., "Quantum Theory of Angular Momentum," Publishing House "Nauka", Leningrad, 1975, pp. 282-285.

[9] M. G. Haines, et al., "Viscous Heating at Stagnation in ZPinches," Physical Review Letters, Vol. 96, No. 7, 2006, pp. 075003-075008.

[10] O. M. Burenkov, Y. N. Dolin, P. V. Duday, V. I. Dudin, V. A. Ivanov, A. V. Ivanovsky, G. V. Karpov, et al., "New Configuration of Experiments for MAGO Program," XIV International Conference on Megagauss Magnetic Field Generation and Related Topics, Maui, 14-19 October 2012, pp. 95-99.

[11] S. Fissenko and I. Fissenko, "Method of Forming Stable States of Dense High-Temperature Plasma," PCT Gazette, No. 46, 2005, p. 553. 